\title{
Breastfeeding attitudes among adolescent mothers attending a nutrition breastfeeding support group
}

\author{
Amy Manion*1, Marilyn Wideman ${ }^{2}$, Amanda Tutlewski $^{1}$ \\ ${ }^{1}$ Rush University, United States \\ ${ }^{2}$ Kaplan University, United States
}

Received: June 17, 2017

Accepted: October 19, 2017

Online Published: October 26, 2017

DOI: $10.5430 /$ cns.v6n1p28

URL: https://doi.org/10.5430/cns.v6n1p28

\begin{abstract}
Objective: Breastfeeding is an ideal way of providing infant nutrition. The aim of this study was to evaluate the effect of breastfeeding and nutrition education on breastfeeding attitudes using a support group model.

Methods: Design: A descriptive comparative design was used to achieve the aim of this study. The study was conducted at a public school for pregnant and/or parenting young women located in a large metropolitan area. Participants: Pregnant or parenting female adolescents $(\mathrm{n}=67)$ in $6^{\text {th }}$ through $12^{\text {th }}$ grade attending a nutrition breastfeeding support group. Instruments: Participants completed an evaluation survey and a breastfeeding questionnaire to assess the effectiveness of the support group and generate information on the group's breastfeeding attitudes and nutrition knowledge.

Results: Breastfeeding attitudes improved as more support group meetings were attended. Public embarrassment due to breastfeeding for new group members was reported at $27 \%$ and as more meetings were attended dropped to zero with a significant likelihood ratio of .013. Breastfeeding attitude questions regarding never being able to give a bottle, being tired about hearing about breastfeeding, thinking breastfeeding is nasty, and hearing that breastfeeding is good all showed positive trends in breastfeeding attitudes.

Conclusions: Attendance at a nutrition breastfeeding support group positively correlated to improved breastfeeding attitudes among adolescent mothers. Peer involvement in a support group environment is an effective intervention for improving breastfeeding knowledge and attitudes in the adolescent population.
\end{abstract}

Key Words: Adolescent mothers, Breastfeeding, Nutrition, Support group, Breastfeeding attitudes

\section{INTRODUCTION}

Breast milk is the ideal food for infants. ${ }^{[1,2]}$ The American Academy of Pediatrics recommends exclusive breastfeeding for the first six months of life, followed by continued breastfeeding in addition to complementary foods for the first 12 months or longer as jointly desired by mother and infant. ${ }^{[3]}$ In the 1970's, the breastfeeding rates in the United States reached a nadir of $22 \% .{ }^{[4]}$ Since then the breastfeeding rates have been steadily rising. Results from the National
Health and Nutrition Examination Survey, 1999-2006 revealed a $17 \%$ overall increase in breastfeeding rates over the last decade, bringing the current rate to $77 \% .^{[5]}$

Although the Healthy People 2010 breastfeeding initiation goal of $75 \%$ has been successfully achieved, breastfeeding rates at 6 and 12 months of age have remained below goal. ${ }^{[6]}$ In 2010, 49\% of infants born in the U.S. were breastfeeding at 6 months and $27 \%$ at 12 months, which is an increase from 2000 when only $35 \%$ of infants were breastfeeding at 6

*Correspondence: Amy Manion; Email: Amy_Manion@rush.edu; Address: Rush University, United States. 
months and $16 \%$ at 12 months. ${ }^{[7]}$ Due to the steady increase seen in breastfeeding rates, the Healthy People 2020 target goals for breastfeeding were increased to $81.9 \%$ for initiation of breastfeeding, $60.6 \%$ of infants breastfeeding at 6 months, and $34.1 \%$ at 12 months. ${ }^{[8]}$

\subsection{Breastfeeding rates and health disparities}

Nevertheless despite a nationwide increase in breastfeeding, the United States has the highest number of adolescent births and the lowest number of adolescent mothers breastfeeding among industrialized countries. ${ }^{[9]}$ In 2015 there were 229,715 live births reported for mothers age 15 to 19 years old. ${ }^{[10]}$ Repeat pregnancies are common among adolescents under 18 years of age, with $30 \%$ to $50 \%$ birthing a second child within 12 to 24 months from the first. ${ }^{[11]}$ Additionally, younger mothers under age 20 are less likely to breastfeed $(43 \%)$ compared to mothers over $20(65 \%) .^{[5,12]}$

Along with dissimilarities in breastfeeding rates based on maternal age, breastfeeding rates differ by socioeconomic characteristics as well. Breastfeeding rates are lowest among women with lower income (57\%) compared to higher income $(74 \%) .{ }^{[5]}$ For adolescent pregnancies, 59\% occur in household with incomes below $200 \%$ of the poverty line, which is reflected in the lower breastfeeding rates for that population range. ${ }^{[13]}$
In addition to socioeconomic characteristics, breastfeeding rates differ by ethnicity. Breastfeeding rates among African American young women who reside in low socioeconomic urban settings are dramatically lower than any other racial group. ${ }^{[14]}$ Mexican-American infants born to mothers under age 20 reported the highest rates of breastfeeding $(66 \%)$ compared to non-Hispanic white (40\%) and non-Hispanic black (30\%). ${ }^{[5]}$ An analysis of data from the Community and Child Health Network by McKinney and associates revealed that Spanish-speaking Hispanic mothers were more likely to initiate breastfeeding and have a positive maternal family history of breastfeeding compared to whites and non-Hispanic black. ${ }^{[15]}$ Further research has demonstrated that not only do racial and ethnic disparities exist but they are further confounded by the lack of implementation of the Ten Steps to Successful Breastfeeding recommended as part of the Baby Friendly Hospital Initiative endorsed by the CDC. ${ }^{[16,17]}$ Table 1 lists the Ten Steps to Successful Breastfeeding. ${ }^{[17]}$ A study by Lind and associates revealed that in areas with greater than $12.2 \%$ black residents, there were large variations in facilities regarding implementation of the recommended maternity practices known to support breastfeeding including early initiation of breastfeeding, rooming-in, and limited use of pacifiers, which further promotes the widening of the gap between breastfeeding initiation and duration rates currently seen between racial and ethnic groups. ${ }^{[18]}$

Table 1. Ten steps to successful breastfeeding

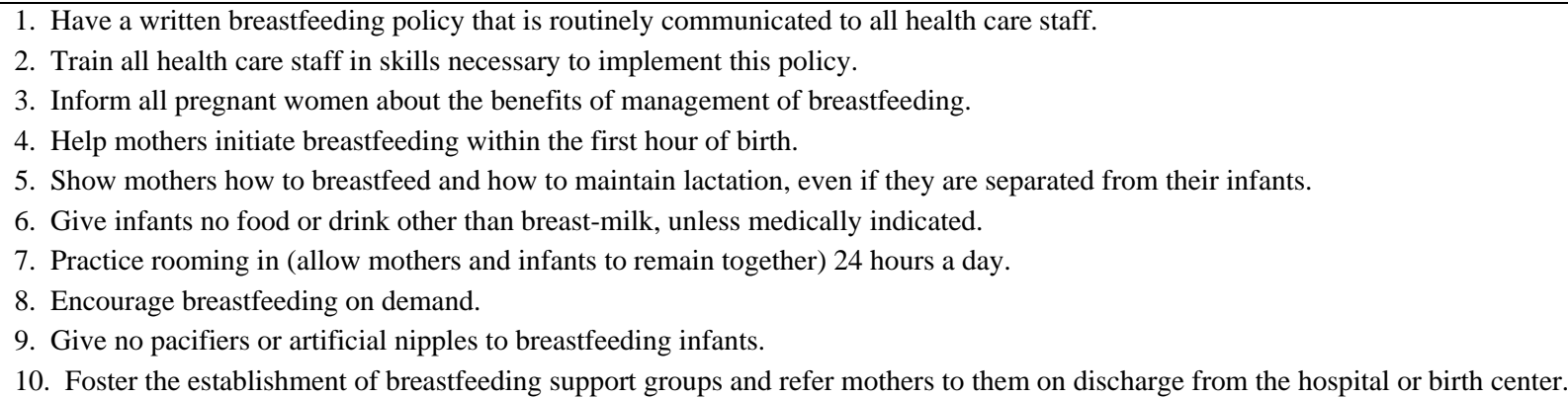

Note. WHO/UNICEF, Ten Steps to Successful Breastfeeding ${ }^{[17]}$

Breastfeeding ethnic and socioeconomic disparities in the adolescent population are alarming given the reported benefits of breastfeeding for the infant as well as the mother. The benefits of breastfeeding for the mother include economic savings, improved maternal bonding, and decreased risk for breast and ovarian cancer. ${ }^{[19]}$ Breastfeeding medical benefits for the infant are profound and include improved gastrointestinal function and host defense, decreased acute illnesses such as otitis media and bronchiolitis, lowered risk of sudden infant death syndrome (SIDS) and necrotizing enterocolitis (NEC), and lowered rates of obesity, asthma, and atopic dermatitis. ${ }^{[3]}$ Infants born to adolescent mothers are not only less likely to breastfeed but also more likely to have nutritional concerns, low birth weight, prematurity, and decreased overall health. ${ }^{[12]}$

\subsection{Breastfeeding barriers and support}

Despite the benefits for both mother and infant, adolescent breastfeeding rates continue to rank below the Healthy People 2020 target goals. ${ }^{[8]}$ Effective interventions, which improve breastfeeding initiation and duration among the vulnerable adolescent mother population, are critically needed. ${ }^{[20]}$ Reported barriers to breastfeeding among adolescent women include fear of pain, public humiliation, lack of interest, ap- 
prehension with the act of breastfeeding, and the perception that breastfeeding will limit their social activity. ${ }^{[21,22]}$ Positive factors influencing breastfeeding among adolescents include wanting to prove they are a "good" mother, knowledge of the benefits of breastmilk, and support from others including peers, family members, and partners. ${ }^{[21]}$ Social support has been proven to be a strong predictor of breastfeeding intention as well as breastfeeding duration. ${ }^{[23-25]}$ Effective social support strategies to promote breastfeeding among adolescents include the use of peer counselors and lactation consultants, inclusion of family in breastfeeding education, and breastfeeding classes. ${ }^{[25]}$ A randomized controlled study of breastfeeding support and education by Wambach and associates found prenatal classes taught by lactation consultants and peer counselors with follow up phone calls to be an effective intervention for increasing breastfeeding duration $(p<.001)$ among adolescent mothers. ${ }^{[25]}$ A systematic literature review of lactation education and support programs reported breastfeeding interventions that included lactation consultants and lactation counselors demonstrated overall increases in breastfeeding rates including initiation. ${ }^{[26]} \mathrm{A}$ breastfeeding infant nutrition support group is one such social strategy.

\subsection{Purpose}

The purpose of this study was to promote healthy infant feeding practices by improving early infant nutrition knowledge, improve breastfeeding attitudes and remove barriers to breastfeeding among pregnant and parenting adolescent women through breastfeeding and nutrition education using a support group model.

\subsection{Aim of the study}

The aim of this study was to evaluate the effect of breastfeeding and infant nutrition education provided to pregnant or parenting adolescent women during a support group setting on breastfeeding attitude, early infant nutrition knowledge and breastfeeding barriers.

\subsection{Research hypothesis}

(1) Breastfeeding education provided during a support group setting has a significant effect on improving breastfeeding attitudes.

(2) Infant nutrition education provided during a support group setting has a significant effect on improving early infant nutrition knowledge.

(3) Providing breastfeeding education will decrease perceived breastfeeding barriers and increase breastfeeding rates in the school setting.

\section{STUDY DESIGN}

\subsection{Sample}

The Food and Fun Fridays program took place at a public school for pregnant and/or parenting young women located in a large metropolitan area. The school has a day care facility on site and a school based health center. A total of 145 female students were enrolled at the time of this study. The students ranged from 13 to 19 years of age and were predominantly African American $(81 \%, \mathrm{n}=118)$, as well as Hispanic/Latino $(17 \%, \mathrm{n}=25)$, multi-racial $(1 \%, \mathrm{n}=1)$, and white $(1 \%, \mathrm{n}=1)$. The majority of students attending the school live in urban high poverty areas with $99 \%$ covered by Medicaid and $96 \%$ qualifying for free or reduced price lunch program. The overall percentage of people living below the poverty level for this community is $45 \%$, more than double the overall poverty rate for the metropolitan area of where this program took place. Institutional Review Board (IRB) approval was obtained from Rush University prior to the start of this study. Informed consent was obtained from all participants. Confidentiality was maintained throughout the study. In addition, all Health Insurance Portability and Accountability Act (HIPAA) regulations were followed. Convenience sampling was used to select participants.

\subsection{Method}

The Food and Fun Fridays program met twice a month during lunchtime under the direction of an interprofessional team, which included dieticians, breastfeeding peer counselors and nurse practitioner students led by an advanced practice nurse. Students voluntarily participated in the program. Informed consent procedures for independent adolescents were followed. There were two daily lunch periods lasting 30 minutes each. Each of the lunchtime groups met for 9 months over the regular school year term for a total of 16 sessions. During the sessions, a broad range of topics was covered regarding pregnancy, parenting, infant nutrition, and breastfeeding. Each lunchtime group received the same information. Examples of sessions conducted by the interprofessional team include: breastfeeding peer counselors demonstrated how to use a breast pump and techniques for breastfeeding success; dieticians discussed overall nutrition including the importance of prenatal nutrition, proper nutrition while breastfeeding, and the vitamins and minerals found in certain foods; and nursing and advanced practice nursing students used games such as "breastfeeding bingo" and "nutrition jeopardy" to encourage interactive student participation while educating on the importance of breastfeeding and nutrition. To measure the effectiveness of the support group and generate information on the group participant's breastfeeding attitudes and nutrition knowledge a breastfeeding attitude questionnaire (BAQ) and an evaluation survey 
were administered. The questionnaire and survey were distributed on three separate occasions throughout the school year- beginning of year, mid-year, and end of school year.

\subsection{Breastfeeding attitudes questionnaire}

The breastfeeding questionnaire used for this study was an adaptation of the BAQ used by Baisch, Fox, and Goldberg involving low-income, pregnant adolescents. ${ }^{[27]}$ The adapted BAQ consisted of a total of 18 items. The first two items on the questionnaire asked "How were you fed as a baby?" and "Where did you hear about breastfeeding?", these items permitted more detailed responses with multiple answers and were analyzed separately. The remaining 16 items were rated using a 3-point Likert scale where 1 indicates "agree", 2 indicates "unsure", and 3 indicates "disagree". The 16 BAQ items were both positive (e.g., "I think breast feeding is good for my baby") and negative (e.g., "I think breast feeding is nasty") with negative items being reversed scored. ${ }^{[28]}$ Total attitude scores range from 16 to 48 , with higher scores representing a more positive breastfeeding attitude. ${ }^{[28]}$

\subsection{Evaluation survey}

The participants completed an evaluation survey consisting of 10 items. The first three items asked if it was their first time attending the support group meeting, how many meetings attended total, and the ages of their children if applicable. Four additional items asked about basic infant nutrition including when to start solids foods. The nutrition questions were selected based on information presented in the group sessions. The remaining three items asked for participants to circle words describing the session they attended that day, and if they would change anything about the session. The survey was based on evaluation methods recommended by The Center for Teen Empowerment. ${ }^{[29]}$

The questionnaire and evaluation survey were both anonymous and individual students were not tracked throughout the program. This was done due to the fluctuation in individual attendance throughout the school year since many students would return to their original school after delivery of their infant. Therefore, data was gathered and analyzed based on how many meetings the participants self-reported they attended as recorded on their evaluation form.

\subsection{Theoretical framework}

For this study social support was defined as the network accessible to an individual through social ties to family, friends, neighbors, and the larger community. ${ }^{[30]}$ The study was guided by House's theory of social support, which includes the categories of informational, instrumental, emotional, and appraisal. ${ }^{[31]}$ Informational support encompassed providing information and advice including discussions on the benefits

Published by Sciedu Press of breastfeeding. Instrumental support included instructions on how to properly clean and use a breast pump. Emotional support involved listening to students' concerns and showing respect to other members attending the group sessions. Offering encouragement and giving feedback provided appraisal support. Other studies involving breastfeeding adolescent mothers have used similar categories of support using House's theory of social support and have reported positive results. ${ }^{[32]}$ A study by Pentecost and Grassley, using House's theory of social support, examined 90 adolescent mothers initiating breastfeeding and found adolescents benefited from a combination of informational, instrumental, emotional and appraisal support. ${ }^{[32]}$

\section{RESULTS}

Data was analyzed using SPSS software and two-tailed Pearson correlation. A total of 60 participants completed the BAQ and 67 completed the evaluation survey. Breastfeeding attitudes improved as more support group meetings were attended. Not feeling embarrassment towards breastfeeding in public for new group members was reported at $27 \%$ $(n=22 / 31)$ and as more meetings were attended dropped to zero with a significant likelihood ratio of .013. Breastfeeding attitude questions regarding never being able to give a bottle, being tired about hearing about breastfeeding, thinking breastfeeding is nasty, and hearing that breastfeeding is good all showed positive trends in breastfeeding attitudes with more frequent group attendance. Nutritional knowledge of having to "eat differently" while breastfeeding was reported at 63\% ( $n=19 / 30)$ for new group members and increased as more meetings were attended to $100 \%$ with a significant likelihood ratio of .010 .

A positive trend was similarly seen regarding lack of breastfeeding knowledge. Initially $61 \%(n=19 / 31)$ of first time participants reported confidence in breastfeeding knowledge. Predictably, as more meetings were attended confidence in breastfeeding knowledge increased to $87 \%(n=7 / 8)$, yet did not reach statistical significance. Another trend was seen regarding influence of the adolescents' mothers and friends negative attitudes towards breastfeeding and attendance at meetings. Participants who described their peers and own mothers as being negative towards breastfeeding attended more meetings.

Of the 60 completed breastfeeding questionnaires, the majority $(48.3 \%, \mathrm{n}=29 / 60)$ recounted having been exclusively bottle fed as an infant, in comparison, to only $8.3 \%$ $(n=5 / 60)$ reporting being exclusively breast-fed. Participants reported that the information that they had received regarding breastfeeding had come mainly from health care providers and family. None of the participants related learning about 
breastfeeding from friends.

The evaluation survey results were overwhelmingly positive. All 67 of the participants completed the survey. Results of the evaluation survey showed that nutrition knowledge improved based on the number of meetings attended. $75 \%(\mathrm{n}=50)$ compared to $25 \%(\mathrm{n}=17)$ correctly reported that drinking apple juice was similar to eating a candy bar as opposed to eating an apple. Of the respondents that answered incorrectly, an overwhelming number had attended four or less support group meetings $(82 \%, \mathrm{n}=14 / 17)$. In addition to improved nutrition knowledge, many of the adolescent participants stated they enjoyed attending the support group and looked forward to its return the following school year. The following statements were written by the students about the support group: "I love this session", "I love this group", "Great time", "Enjoyful", "Thank you", and "This is a great way to learn about baby things". Furthermore, of the adolescents who gave birth during the school year, 15 girls reported initiation of breastfeeding, with 5 students breastfeeding for 4 months, and 2 students reporting breastfeeding for 6 months or longer. The previous school year none of the recently delivered students reported breastfeeding their infants beyond 4 weeks.

\section{Discussion}

The trend of increased confidence in breastfeeding knowledge with increased support group attendance demonstrates how breastfeeding attitudes can improve by educating young mothers about infant nutrition practices using a support group model. Maternal breastfeeding knowledge, attitudes, and confidence have been shown to be a strong predictor of the method of infant feeding. ${ }^{[28,33]}$ Mossman and associates found a significant correlation between high prenatal confidence and initiation and duration of breastfeeding among adolescent mothers based on initial scores from the BAQ. ${ }^{[28]}$ A more recent study regarding breastfeeding knowledge found a positive correlation between awareness of the World Health Organization's recommendation to exclusively breastfeed for 6 months and breastfeeding initiation and duration rates. ${ }^{[34]}$ Likewise, the effects of the support group intervention on improved breastfeeding attitudes are palpable in the increased number of new breastfeeding mothers reported by the school based health clinic. The rise seen in breastfeeding rates among the students along with increased breastfeeding knowledge confidence supports the assumption that providing breastfeeding education will improve breastfeeding attitudes and lead to increased breastfeeding rates.

There are many barriers to breastfeeding. One major barrier that receives more attention than most is public embarrassment. Stuebe and Bonuck studied low-income women and discovered feeling more comfortable with public breastfeeding and increased knowledge regarding the benefits of breastfeeding were direct predictors of exclusive breastfeeding intention. ${ }^{[33]}$ Similar results are reflected in this study, which demonstrated a decrease in public embarrassment of breastfeeding as more meetings were attended. The embarrassment of breastfeeding in public is a common reported barrier to breastfeeding especially in the adolescent population. ${ }^{[35,36]}$ Furthermore, frequent media reports of mother's being shamed for publicly breastfeeding reinforces the preconceived notion that breastfeeding in public is an uncomfortable experience. An on line poll by Fertility Flower reported $15 \%$ of the women surveyed who were formula feeding their infants stated one of the reasons they chose formula was due to potential embarrassment of breastfeeding in public. ${ }^{[37]}$ The improved attitude results from this study regarding breastfeeding in public demonstrates how increased breastfeeding knowledge can influence feeding choices, decrease modesty concerns with breastfeeding, and potentially reverse the negative message portrayed through the media and in the community.

Other barriers to breastfeeding include lack of social support and limited access to breastfeeding information. Research using focus groups with African American women identified lack of breastfeeding information, logistical struggles with breastfeeding in public, and lack of a support from family and health care providers as barriers to breastfeeding. ${ }^{[2]}$ The results of this study demonstrated similar barriers such as lack of family support and the desire for more information. The positive trend of increased support group attendance among participants, who indicated negative peer and maternal support for breastfeeding, coincides with the reported lack of pre and post partum breastfeeding information young mothers receive from the health care workers, family, and friends. ${ }^{[12,38]}$ Similarly, adolescent mothers have reported receiving meager knowledge regarding the benefits of breastfeeding post partum and only being asked if they were breast or bottle feeding their infant with no further feeding support. ${ }^{[38]}$ Other research has found that adolescents mothers value breastfeeding advice from nursing staff and appreciate discussions about feeding. ${ }^{[32]}$ The increased attendance demonstrated by the participants who reported lack of peer and maternal support is encouraging and reflective of how a support group intervention guided by an interprofessional health care team can provide breastfeeding knowledge to young mothers who are seeking information and support.

In addition to health care team members, peers are another important component of providing breastfeeding support to adolescent mothers. Successful breastfeeding peer counselor programs such as the You Can Do It (YCDI) initiative im- 
plemented by WIC clinics is an example of how peers can be used to support adolescent mothers who choose to breastfeed. ${ }^{[39]}$ A meta-analysis review of eleven controlled studies examining exclusive breastfeeding in low and middle-income countries discovered peer support significantly increased exclusive breastfeeding duration. ${ }^{[40]}$ A study of pregnant African American women ages 18 to 45 found the intent to breastfeed doubled after attending WIC breastfeeding peer support programs. ${ }^{[41]}$ The results from this study validate how a support group intervention can increase breastfeeding knowledge and negate outside barriers to breastfeeding from family and friends by offering peer support to young mothers, leading to increased initiation and duration of breastfeeding.

In addition, the camaraderie atmosphere of the support group cannot be overlooked. Students that attended the group sessions would often break off into smaller groups after the main meeting was over to further discuss breastfeeding and infant care. These conversations built friendships, offered further peer support, and empowered the young women to share their knowledge with each other.

\section{Limitations}

A limitation of this study was the anonymity of the questionnaire results. In the study by Baisch and associates higher BAQ scores was significantly linked to postnatal breastfeeding initiation. ${ }^{[27]}$ A more recent study by Mossman and associates found similar results, with higher BAQ scores predicting increased breastfeeding initiation and confidence among adolescent mothers. ${ }^{[2]}$ Since the questionnaire results were anonymous there was not the opportunity to directly correlate breastfeeding attitude scores and initiation of breastfeeding. Nevertheless, there is the potential to assume that the increased breastfeeding rates for the students is attributed to the higher BAQ scores seen with increased support group attendance over the course of the school year.

\section{Conclusions}

Parenting and breastfeeding is challenging for all mothers. Especially for adolescent mothers, the benefits of breastfeeding can be lost in the daily struggle to attend school and parent a child. Peers can offer support and knowledge to young mothers. The results of this study demonstrate a positive correlation between improvements in breastfeeding attitudes and support group involvement as an effective intervention for improving breastfeeding attitudes, decreasing breastfeeding barriers, increasing breastfeeding rates, and improving early infant nutrition knowledge in the adolescent population.

\section{Nursing implications and recommendations}

Nurses are in a unique position to influence infant nutrition attitudes and practices by providing knowledge of the positive benefits of breastfeeding for both the mother and infant. Nurses are in a unique position since they are often the first person to offer hands on support and in the moment advice to young adolescent mothers initiating breastfeeding. Nurses can further assist young mothers with their decisions to breastfeed by assuring the Ten Steps to Successful Breastfeeding are properly implemented with each young mother who delivers at their institution. In addition, nurses should encourage and foster the use of lactation consultants and peer counselor services. Further research is needed to explore more opportunities to effectively engage with young mothers and bolster their social support networks.

\section{CONFLicts OF INTEREST Disclosure}

The authors declare they have no conflicts of interest.

\section{REFERENCES}

[1] Jones JR, Kogan MD, Singh GK, et al. Factors associated with exclusive breastfeeding in the United States. Pediatrics. 2011; 128(6): 1117-1125. https://doi.org/10.1542/peds . 2011-0841

[2] Lewallen LP, Street DJ. Initiating and sustaining breastfeeding in African American women. Journal of Obstetric, Gynecologic, \& Neonatal Nursing. 2010; 39: 667-674.

[3] American Academy of Pediatrics (AAP). Breastfeeding and the use of human milk. Pediatrics. 2012; 129(3): e827-e841. PMid: 22371471. https://doi.org/10.1542/peds.2011-3552

[4] Philipp BL, Sheina J. African American Women and Breastfeeding, The Courage to Love: Infant Mortality Commission. Joint Center for Political and Economic Studies Health Policy Institute: Washington DC; 2007

[5] McDowll MA, Wang CY, Kennedy-Stephensen J. Breastfeeding in the United States: Findings from the National Health and Nutrition Examination Survey, 1999-2006. NCHS Data Brief, no. 5. Hyattsville,
MD: National Center for Health Statistics; 2008. Available from: http://www.cdc.gov/nchs/data/databriefs/db05.htm

[6] Center for Disease Control and Prevention. Breastfeeding Report Card - United States 2010. 2010. Available from: http://www.cdc.gov/breastfeeding/pdf/Breastfeedin gReportCard2010.pdf

[7] Center for Disease Control and Prevention (CDC). Breastfeeding Report Card- United States 2013. 2013. Available from: http://www.cdc.gov/breastfeeding/pdf/2013brea stfeedingreportcard.pdf

[8] Healthy People 2020. Available from: http://www . healthypeo ple.gov/2020/topicsobjectives2020/objectiveslist.a spx?topicId $=26$

[9] Feldman-Winter L, Shaikh U. Optimizing breastfeeding promotion and support in adolescent mothers. Journal of Human Lactation. 2007; 23: 362-367. PMid: 17991802. https://doi.org/10.117 $7 / 0890334407308303$ 
[10] Matrin JA, Hamiltion BE, Osterman MJK, et al. Births: Final data for 2015. National Vital Statistics Report. 2017; 66(1): 1-70. https : //www.cdc.gov/nchs/data/nvsr/nvsr66/nvsr66_01.pdf

[11] Holness N. A global perspective on adolescent pregnancy. International Journal of Nursing Practice. 2015; 21: 677-681. PMid: 24674421. https://doi.org/10.1111/ijn.12278

[12] Apostolakis-Kyrus K, Valentine C, DeFranco E. Factors associated with breastfeeding initiation in adolescent mothers. The Journal of Pediatrics. 2013; 163: 1489-94. PMid: 23896187. https: //doi.org/10.1016/j.jpeds.2013.06.027

[13] Petry R. Breastfeeding and socioeconomic status: An analysis of breastfeeding rates among low-SES mothers. Poverty and Human Capability Studies Capstone. 2013; 1-38. Available from: https://www. shepherdconsortium.org/wp-content /uploads/2013/04/Petry.2013.POV-423-Capstone.pdf

[14] Tucker CM, Wilson EK, Samandari G. Infant feeding experiences among teen mothers in North Carolina: Findings from a mixedmethods study. Int Breastfeed J. 2011; 6: 6-14. PMid: 21955654 https://doi.org/10.1186/1746-4358-6-14

[15] McKinney CO, Hahn-Holbrook J, Chase-Lansdale PL, et al. Racial and ethnic differences in breastfeeding. Pediatrics. 2016; 138(2): e20152388. PMid: 27405771. https://doi.org/10.1542/peds .2015-2388

[16] Centers for Disease Control and Prevention. Strategies to Prevent Obesity and Other Chronic Diseases: The CDC Guide to Strategies to Support Breastfeeding Mothers and Babies. Atlanta: U.S. Department of Health and Human Services; 2013. Available from: https : //www.cdc.gov/breastfeeding/pdf/bf-guide-508.pdf

[17] WHO/UNICEF. Ten Steps to Successful Breastfeeding. Available from: http://www. tensteps .org

[18] Lind JN, Perrine CG, Li R, et al. Racial disparities in access to maternity care practices that support breastfeeding- United States, 2011. Morbidity and Mortality Weekly Report. 2014; 63(3): 725-728. PMid: 25144543.

[19] Burns C, Dunn A, Brady M, et al. Pediatric primary care (5th ed.). St. Louis, MO: Saunders; 2012.

[20] Wambach KA, Cohen SM. Breastfeeding Experiences of Urban Adolescent Mothers. Journal of Pediatric Nursing. 2009; 24(4): 244254. PMid: 19632502. https://doi.org/10.1016/j.pedn.2 008.03 .002

[21] Kanhadilok S, McGrath JM. An Integrative review of factors influencing breastfeeding in adolescent mothers. The Journal of Perinatal Education. 2015; 24(2): 119-127. PMid: 26957895. https : //doi.org/10.1891/1946-6560.24.2.119

[22] Nelson AM. Adolescent attitudes, beliefs, and concerns regarding breastfeeding. American Journal of Maternal Child Nursing. 2009; 34(4): 249-55. PMid: 19587570. https ://doi .org/10.1097/01 .NMC.0000357918.18229.25

[23] Sipsma HL, Magriples U, Divney A, et al. Breastfeeding behavior among adolescents: initiation, duration, and exclusivity. Journal of Adolescent Health. 2013; 53(3): 394-400. PMid: 23725911 https://doi.org/10.1016/j.jadohealth.2013.04.005

[24] Bibbins-Domingo K, Grossman DC, Curry SJ, et al. Primary care interventions to support breastfeeding: US Preventative Services Task Force Recommendation Statement. JAMA. 2016; 316(16): 16881693. PMid: 27784102. https://doi.org/10.1001/jama.201 6.14697

[25] Wambach KA, Aaronson L, Breedlove G, et al. A randomized controlled trial of breastfeeding support and education for adolescent mothers. Western Journal of Nursing Research. 2011; 33(4): 486505. PMid: 20876551. https ://doi.org/10.1177/0193945910 380408
[26] Patel S, Patel S. The Effectiveness of lactation consultants and lactation counselors on breastfeeding outcomes. Journal of $\mathrm{Hu}$ man Lactation. 2016; 32(3): 530-541. PMid: 26644419. https: //doi.org/10.1177/0890334415618668

[27] Baisch MJ, Fox RA, Goldberg BD. Breast-feeding attitudes and practices among adolescents. Journal of Adolescent Health Care. 1989; 10(1): 41-45. https://doi.org/10.1016/0197-0070(89) 900 46-6

[28] Mossman M, Heaman M, Dennis C, et al. The Influence of adolescent mothers' breastfeeding confidence and attitudes on breastfeeding initiation and duration. Journal of Human Lactation. 2008; 24(3): 268-277. PMid: 18689714. https ://doi.org/10.1177/089033 4408316075

[29] Pollack S, Fusoni M. Moving Beyond Icebreakers: An Innovative Approach to Group Facilitation, Learning, and Action. The Center for Teen Empowerment, Inc.; Boston, MA; 2005.

[30] Ozbay F, Johnson DC, Dimoulas E, et al. Social support and resilience to stress. Psychiatry. 2007; 4(5): 35-40. PMid: 20806028.

[31] House JS. Work Stress and Social Support. Reading, MA; Addison Wesley; 1981.

[32] Pentecost R, Grassley JS. Adolescents' needs for nurse support when initiating breastfeeding. Journal of Human Lactation. 2014; 30(2): 224-228. PMid: 24194610. https ://doi.org/10.1177/089033 4413510358

[33] Stuebe AM, Bonuck K. What predicts intent to breastfeed exclusively? Breastfeeding knowledge, attitudes, and beliefs in a diverse urban population. Breastfeeding Medicine. 2011; 6(6): 413-420. PMid: 21342016. https://doi .org/10.1089/bfm. 2010.0088

[34] Wen LM, Simpson JM, Rissel C, et al. Awareness of breastfeeding recommendations and duration of breastfeeding: findings from the Healthy Beginnings Trial. Breastfeeding Medicine. 2012 Aug; 7: 223-9. PMid: 22568472. https://doi.org/10.1089/bfm. 2011 .0052

[35] McFadden A, Toole G. Exploring women's views of breastfeeding: a focus group study within an area with high levels of socioeconomic deprivation. Maternal \& Child Nutrition. 2006; 2(3): 156168. PMid: 16881928 . https ://doi.org/10.1111/j.1740-870 9.2006.00054.x

[36] Tenfelde S, Finnegan L, Hill PD. Predictors of breastfeeding exclusivity in a WIC sample. Journal of Obstetric, Gynecologic, \& Neonatal Nursing. 2011; 40: 179-189. PMid: 21314715. https: //doi.org/10.1111/j.1552-6909.2011.01224.x

[37] Willard L. Poll: Women not breastfeeding because of embarrassment? She Knows Parenting. 2010. Available from: http://www . sheknows. com/parenting/articles/821692/r ecent-poll-discusses-breastfeeding-statistics

[38] Spear H. Breastfeeding behaviors and experiences of adolescent mothers. American Journal of Maternal Child Nursing. 2006; 31: 106-13. PMid: 16523036. https://doi.org/10.1097/000057 21-200603000-00010

[39] Edmunds LS, Furrina FL, Eldridge JD, et al. Outcome evaluation of the You Can Do It initiative to promote exclusive breastfeeding among women enrolled in the New York State WIC program by race/ethnicity. Journal of Nutrition Education and Behavior. 2017; 49(7): S162-S168. PMid: 28689553. https://doi.org/10.101 $6 / j \cdot j n e b .2017 .05 .350$

[40] Sudfeld CR, Fawzi WW, Lahariya C. Peer support and exclusive breastfeeding duration in low and middle-income counties: a systematic review and meta-analysis. PLoS One. 2012; 7(9): e45143. https://doi.org/10.1371/journal.pone.0045143

[41] Mickens A, Modeste N, Montgmery S, et al. Peer support and breastfeeding intentions among black WIC participants. Journal of Human Lactation. 2009; 25(2): 157-162. 